\title{
Hypertension in elderly: A study of hypertension in epigenetic perspective
}

\author{
Nurin Nadzifatil Fitriyah ${ }^{1}$, Indah Mardiana ${ }^{2}$ \\ 1) Departement of Biomedic, Faculty of Medicine and Health, Universitas Muhammadiyah Jakarta \\ 2) Medical Study Program, Faculty of Medicine and Health, Universitas Muhammadiyah Jakarta \\ *nurin.n.fitriyah@gmail.com
}

\begin{abstract}
Background: as is known, epigenetic events are defined as modifications in phenotype or gene expression, which caused by interactions between genes and the environment without changing the order of their nitrogenous bases. This article will discuss the role of epigenetics in one of the degenerative diseases that are often found in the elderly, namely hypertension. In this review article, we will mainly discuss the events of histone modification and DNA methylation, for example, DNA methylation in the $\mathrm{CpG}$ islands and the acetylation of histone pathway are identified to limit the development of nephrons, thus opening up to lhypertension-related with exposure to high salt diets. Results: lack of maternal water and protein deficiency have been shown to increase the appearance of the renin-angiotensin system gene in the children. Conclusion: these results indicate targets for the interaction of environmental genes in various conditions of hypertension and essential hypertension.
\end{abstract}

Keywords: Hypertension, Elderly, Epigenetics.

\section{INTRODUCTION}

A situation of high blood pressure (BP) in blood vessels that carry blood from the heart to body tissues called hypertension. For several years, hypertension definite as a BP reading of 140/90 $\mathrm{mm} \mathrm{Hg}$ or higher in adults. But, the American College of Cardiology / American Heart Association (ACC/AHA) suggestions additional guidance on BP reading of 130/80 $\mathrm{mm} \mathrm{Hg}$ or higher. Furthermore, hypertension remains the main risk factor for several diseases, such as myocardial infarction, heart failure, end-stage renal disease, and stroke (1).

Based on the study, it has known that several chronic conditions can increase the hypertension risk, such as aging, smoking, obese, harmful eating habits, and secondary diseases others such as chronic kidney disease (CKD), family genetic history, diabetes mellitus, obstructive sleep apnea, and psychosocial stress (1).

Hypertension was identified to ensue, one of which is due to epigenetic mechanisms. Epigenetic mechanisms have a role in the regulation of physiology and disease by changing gene expression, which is the effect of interactions between DNA and environmental factors. This change due to the epigenetic result is considered to have a more stable and continuing effect (2). 


\section{LITERATURE REVIEW}

\section{Definition of Epigenetic}

Epigenetics is the mechanism of an individual's phenotypic changes that are not caused by variations in the sequence of DNA nitrogen bases. There are several types of epigenetic events, including DNA methylation, histone modification, and chromatin remodeling. This article discusses two epigenetic events that affect hypertension, they are DNA methylation and histone modification. It has known that epigenetic from gene expression caused by changes in DNA methylation and histone modification can reduce the number of stem cells directed to kidney disease and hypertension (3).

\section{DNA Methylation}

The addition of the methyl Sadenosylmethionine (SAM) donor group to a position of the fifth carbon (C5) of the cytosine residue pathway contained in 5'G-phosphate-C-3 '(CpG) dinucleotides defined as DNA methylation. $\mathrm{CpG}$ dinucleotides bind to guanine via phosphodiester bonds and can be created as a group, and also consist of cytosines called CpG islands (CGI). CGI is linked to regional promoters and regulations in initiation transcription $(4,5)$. We can see the Biochemical structure in figure 1.
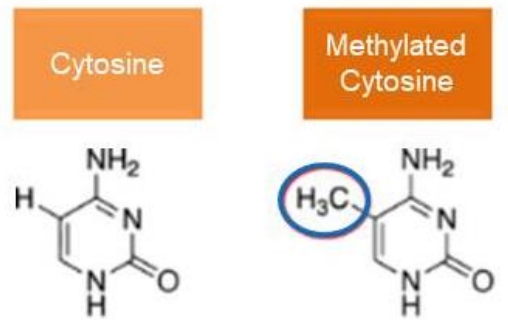

Figure 1. Adding methyl group on cytosine.

DNA methyltransferase (DNMT) enzyme-catalyzed DNA methylation. While DNA demethylation enzyme- catalyzed DNA demethylation. DNA methylation is completed by adding a methyl group to DNA. In mammals, there are three types of DNMT: i) DNMT1, which preserving methylation, ii) DNMT3a and iii) DNMT3b is de novo methyltransferase that methylates the DNA genome during early embryonic development (6).

DNA methylation controls gene expression by inhibiting interactions between transcription factors into DNA. DNA methylation suppressed the structure of chromatin, so the transcription factors do not have contact with DNA (7).

\section{DNA methylation of hypertension}

Several studies have explained the mechanism of epigenetics in hypertension. The involvement of the Renin-Angiotensin System (RAS) in the regulation of arterial pressure the effect of epigenomic regulation of the RAS has been extensively tested in animal models of systemic hypertension (8).

Renin-Angiotensin System (RAS) is one of the main controls on systemic blood pressure, which can contribute to systemic hypertension in humans by overstating the vasopressor response to drugs, nutrition, or exercise (9). For example, it has been shown that the angiotensin $1 \alpha$ receptor (AT1aR). The angiotensin $1 \alpha$ receptor is encoded by Atgr1 $\alpha$, and significantly increased in spontaneous hypertensive mice (SHR) compared to its complement, Wistar-Kyoto (WKY) mice, which may be responsible for BP increases in SHR (10).

$$
\text { Besides, }
$$

hypomethylation angiotensin type II receptor gene promoters 1 (AT1bR), (Atgr1 $\beta)$, in the adrenal glands of maternal low-protein mice show hypertension in response to salt intake (9). It was reported in other studies 
that the proximal promoter of the angiotensin AT1b receptor gene in the adrenal glands of mice was significantly reduced, and the expression of the AT1b gene was highly dependent on methylation of the promoter. When the expression of the AT1b gene in the adrenal gland is controlled by hypomethylation through the first week of life, increased expression of this receptor protein increases the adrenal gland's response to angiotensin. It can contribute to an excessive reaction by epigenetic mechanisms (11).

It has been reported that exposure to prenatal protein deficiency can increase the expression of angiotensinogen mRNA and angiotensin-converting enzymes, by decreasing levels of angiotensin II receptor mRNA.(12) Another study, which uses 3 days of water shortages in pregnant mice, reported that an increase in plasma sodium concentration and osmolality was related to an increase in fetal liver mRNA and fetal angiotensinogen and plasma angiotensin I plasma and plasma angiotensin II plasma (9).

Moreover, blood pressure after angiotensin II administration increases and baroreflex sensitivity is attenuated in adolescents from water-deficient mothers, although there is no effect on basal blood pressure, Cardiac angiotensin receptor mRNA and protein expression are also higher in water-deficient mothers and offspring (9).

These findings indicate that shortterm maternal dehydration can have a profound and persistent impact on fetal RAS genotypes that seem to have the capacity to respond responsively to vasopressin responsiveness in adolescents and adults. Although specific epigenetic mechanisms are not identified for maternal water deficiency, aims (other than the RAS gene) may be osmolality and sodium transporters that are sensitive to furosemide. Protein, which appears to be responsible for the high sodium permeability reported in human erythrocytes with a genetic predisposition to salt-sensitive hypertension (13).

The expression of angiotensinogen protein fixed, but angiotensin-converting enzyme protein and angiotensin receptor protein have decreased. These changes are related to the hypomethylation of the $\mathrm{CpG}$ islands in the angiotensin-converting enzyme promoter area and the upregulation of various microRNA sequences, short noncoded RNAs that regulate mRNA translation of the angiotensin converter translation enzyme (12)

From the study, it was known that hypomethylation of the RAS gene, for example, the angiotensin-converting enzyme gene, could contribute to an increase in the number of circulating vasoconstrictors and various hypertensive conditions, and cognitive deficits related with essential hypertension (12).

\section{Histon modification}

To form DNA, it needs modified histones. As we can see in figure 2, the strands of DNA are bound over octamer two subunits per each core histone ( $\mathrm{H} 2 \mathrm{~A}, \mathrm{H} 2 \mathrm{~B}, \mathrm{H} 3$ and H4) and are linked by connecting histones (H1). That form is forming the nucleosomes. The $\mathrm{N}$-terminal histone tail contains amino acid residues. It can be affected by several types of histone modification, such as methylation, acetylation, ubiquitylation, phosphorylation, and sumoylation. 


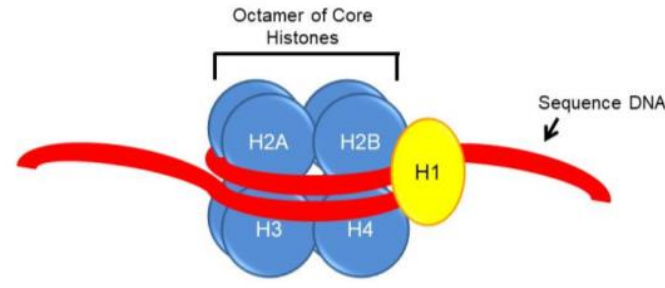

Figure 2. The Structure of Histone

Histone methyltransferase (HMTase) organized the addition of methyl groups to the lysine $(\mathrm{K})$ and arginine $(\mathrm{R})$ residues. It called histone methylation. Histone methylation is correlated to gene silencing, depending on the position of the methylated residue that will suppress gene expression (4). Instead, methylation of lysine 4 residue in $\mathrm{H} 3$ ( $\mathrm{H} 3 \mathrm{~K} 4)$ leads to gene expression (14).

The transfer of acetyl groups from acetyl-CoA to $\varepsilon-\mathrm{NH} 2$ clusters of $\mathrm{N}$ terminal histone residues catalyzed by the enzyme histone acetyltransferase (HAT). It defined as histone acetylation. It will activate gene expression and histone enzyme deacetylase (HDAC) which has a role in the process of deacetylation of histones. It will prevent gene expression (15).

Then, the phosphorylation of histones is the addition of phosphate groups to histone proteins. It follows in serine residues and generally leads to gene activation (16).

Histone ubiquitylation is the addition of the ubiquitin group to histone proteins. Ubiquitin is a polypeptide consisting of 76 amino acids that can be enzymatically attached to all kinds of proteins.

Thus, sumoylation is a modification of histone. It attached to a small modifier associated with ubiquitin-protein (SUMO). Modification of histone by sumoylation can cause gene expression and inhibit other types of histone modification (17).

\section{Histon modification of hypertension}

It has known from the results of studies, that in addition to regulation of DNA methylation in RAS, the SHR aorta also shows higher $\mathrm{H} 3 \mathrm{Ac}$ and $\mathrm{H} 3 \mathrm{~K} 4 \mathrm{me} 3$ enrichment, while $\mathrm{H} 3 \mathrm{~K} 9 \mathrm{me} 2$ improvement is reduced in the promoter of the angiotensin 1 converting enzyme (ACE1). Therefore, the regulation of DNA methylation and the modification of histone occur in the RAS system (18).

Lee et al, have studied that ACE1 is controlled in the local SHR network through modification of the histone code. It is known that histone modification is a main epigenetic factor that increases ACE1 expression in SHR local tissue. Local RAS or tissue RAS, for example, the adrenal glands, aorta, liver and kidney SHR, have higher levels of Ace1 mRNA and protein than WKY mice. The increased regulation of Ace 1 mRNA and protein in these tissues matches with the enrichment of the Ace1 promoter by activating chromatin markers, such as $\mathrm{H} 3 \mathrm{Ac}$ and H3K4me3. The Ace1 promoter from the local SHR network is simultaneously enriched with $\mathrm{H} 3 \mathrm{Ac}$ and $\mathrm{H} 3 \mathrm{~K} 4 \mathrm{me} 3$. Histon acetylation is functionally associated with $\mathrm{H} 3 \mathrm{~K} 4$ methylation. When histone is made hyperacetylated with HDAC inhibitors, both the abundance and the level of methylation of $\mathrm{H} 3 \mathrm{~K} 4$ (monomethylation, dimethylation, and trimethylation) also increase (18).

\section{CONCLUSION}

Epigenetics, in this case DNA methylation and histone modification, has a role in degenerative disease of the elderly including hypertension. 


\section{ACKNOWLEDGMENT}

The author thanks the source of reference in this review article, and the faculty of medicine and health Universitas Muhammadiyah Jakarta.

\section{CONFLICT OF INTEREST}

The author does not have any conflict of interest in writing this article.

\section{REFERENCE}

1. Kotchen TA, Liu Y. Epigenomics of Hypertension. Semin Nephrol. Elsevier; 2013;33(4):392-9.

2. Liang M. Epigenetic mechanisms and hypertension. Hypertension. 2018;72(6):1244-54.

3. Koleganova N, Piecha G, Ritz E. Prenatal Causes of Kidney Disease. Blood Purif. 2009;27:48-52.

4. Rose NR, Klose RJ. Biochimica et Biophysica Acta Understanding the relationship between DNA methylation and histone lysine methylation. Biochim Biophys Acta. Elsevier B.V.; 2014;1839(12):1362-72.

5. Deaton AM, Bird A. CpG islands and the regulation of transcription $\mathrm{CpG}$ islands and the regulation of transcription. Genes Dev. 2011;25:1010-22.

6. Okano M, Xie S, Li E. Cloning and characterization of a family of novel mammalian DNA ( cytosine-5 ) methyltransferases Non-invasive sexing of preimplantation stage mammalian embryos. Nat Genet. 1998;19:219-20.

7. Oh JH, Gertych A, Tajbakhsh J. Nuclear DNA Methylation and Chromatin Condensation Phenotypes Are Distinct Between Normally Proliferating / Aging ,
Rapidly Growing / Immortal , and Senescent Cells. Oncotarget. 2013;4(3):474-93.

8. Wise IA, Charchar FJ. Epigenetic Modifications in Essential Hypertension. Int $\mathbf{J}$ Mol Sci. 2016;17(451):1-14.

9. Shim CY, Ha J, Park S, Choi E, Korea S. Exaggerated Blood Pressure Response to Exercise Is Associated With Augmented Rise of Angiotensin II During Exercise. J Am Coll Cardiol. 2008;52(4):28792.

10. Pei F, Wang X, Yue R. Differential expression and DNA methylation of angiotensin type $1 \mathrm{~A}$ receptors in vascular tissues during genetic hypertension development. Mol Cell Biochem. 2015;

11. Bogdarina I, Welham S, King PJ, Burns SP, Clark AJL. Epigenetic Modification of the ReninAngiotensin System in the Fetal Programming of Hypertension. Circ Res. 2007;100:520-6.

12. Goyal R, Goyal D, Leitzke A, Gheorghe CP, Longo LD. Brain Renin-Angiotensin System: Fetal Epigenetic Programming. Reprod Sci. 2010;17(3):227-38.

13. $\mathrm{Yu} \mathrm{Z}$, Kong Q, Kone BC. CREB trans -activation of disruptor of telomeric silencing-1 mediates forskolin inhibition of CTGF transcription in mesangial cells. Am Physiol Soc. 2020;298(9):617-24.

14. Stein AB, Jalife J, Dressler GR, Stein AB, Jones TA, Herron TJ, et al. Loss of $\mathrm{H} 3 \mathrm{~K} 4$ methylation destabilizes gene expression patterns and physiological functions in adult murine cardiomyocytes. $\mathbf{J}$ Clin Invest. 2011;121(7):2641-50. 
15. Peserico A, Simone C. Physical and Functional HAT / HDAC Interplay Regulates Protein Acetylation Balance. J Biomed Biotechnol. 2011;2011:1-10.

16. Berger SL. Histone modifications in transcriptional regulation. Curr Opin Genet Dev. 2002;12:142-8.

17. Shiio Y, Eisenman RN. Histone sumoylation is associated with transcriptional repression. PNAS. 2003;100(23):13225-30.

18. Lee H, Cho H, Lee D, Kim K, Han HS, Kim IK. Enzyme 1 in Spontaneously Hypertensive Rats Through Histone Code Modifications. Hypertension. 2012;59:621-6. 